\title{
Development and Validation of a Nomogram Prognostic Model for Resected Limited-Stage Small Cell Lung Cancer Patients
}

\author{
Qingpeng Zeng, Jiagen Li, Fengwei Tan, Nan Sun, Yousheng Mao, Yushun Gao, Qi Xue, \\ Shugeng Gao, Jun Zhao, and Jie He \\ Department of Thoracic Surgery, National Cancer Center/National Clinical Research Center for Cancer/Cancer Hospital, \\ Chinese Academy of Medical Sciences and Peking Union Medical College, Beijing, China
}

\begin{abstract}
Background. In this study, we developed and validated nomograms for predicting the survival in surgically resected limited-stage small cell lung cancer (SCLC) patients.
\end{abstract}

Methods. The SCLC patients extracted from the Surveillance, Epidemiology, and End Results database between 2000 and 2014 were reviewed. Significant prognostic factors were identified and integrated to develop the nomogram using multivariable Cox regression. The model was then validated internally by bootstrap resampling, and externally using an independent SCLC cohort diagnosed between 2000 and 2015 at our institution. The prognostic performance was measured by the concordance index $(\mathrm{C}$ index) and calibration curve.

Results. A total of 1006 resected limited-stage SCLC patients were included in the training cohort. Overall, 444 cases from our institution constituted the validation cohort. Seven prognostic factors were identified and entered into the nomogram construction. The $\mathrm{C}$-indexes of this model in the training cohort were $0.723,0.722$, and 0.746 for predicting 1-, 3-, and 5-year overall survival (OS),

Supplementary Information The online version of this article (h ttps://doi.org/10.1245/s10434-020-09552-w) contains supplementary material, which is available to authorized users.

(C) The Author(s) 2021

First Received: 21 October 2020

Accepted: 11 December 2020;

Published Online: 2 March 2021

J. Zhao

e-mail: drzhaojun@126.com

J. $\mathrm{He}$

e-mail: prof.jiehe@gmail.com respectively, and $0.816,0.710$, and 0.693 , respectively, in the validation cohort. The calibration curve showed optimal agreement between nomogram-predicted survival and actual observed survival. Additionally, significant distinctions in survival curves between different risk groups stratified by prognostic scores were also observed. The proposed nomogram was then deployed into a website server for convenient application.

Conclusions. We developed and validated novel nomograms for individual prediction of survival for resected limited-stage SCLC patients. These models perform better than the previously widely used staging system and may offer clinicians instructions for strategy making and the design of clinical trials.

Lung cancer still remains the leading cause of cancerrelated deaths worldwide. Small cell lung cancer (SCLC) accounts for approximately $15-20 \%$ of lung cancer patients, of whom approximately $30 \%$ are non-metastatic at initial diagnosis. ${ }^{1}$ SCLC is characterized by rapid progression, high aggressiveness, and inferior prognosis; multimodality therapy, including chemotherapy and radiotherapy, is still the standard management of this disease. $^{2}$ The role of surgery in the treatment of SCLC is currently considered very limited since the two previous clinical trials ${ }^{3,4}$ showed no survival benefit from the introduction of surgery into the treatment modality; however, several studies have revealed that surgery may achieve favorable survival outcomes in patients with earlystage disease. ${ }^{5,6}$ Currently, the National Comprehensive Cancer Network (NCCN) guidelines recommend surgery for selected cases of clinical stage $T 1-2, N 0$ SCLC. ${ }^{7}$ 
The two-tier staging system (limited disease and extensive disease) introduced by the Veterans Administration Lung Study Group (VALSG) was used as the foundation of the treatment strategy and the major prognostic parameter; however, individual survival differs widely in the same stage. The American Joint Committee on Cancer (AJCC) 7th TNM staging system was reported to contribute to a more precise prognosis and has been adopted for the staging of SCLC. ${ }^{8,9}$ In addition, several previous studies have revealed other independent prognostic factors, including sex, lobectomy, adjuvant chemotherapy, or radiotherapy, for surgically treated SCLC. ${ }^{10-12}$ Hence, based on these above factors, a more individualized prediction of survival could be achieved. Nomogram models have been widely used as a feasible tool to predict individualized prognosis for cancer patients, which could benefit treatment strategy making and clinical trials.

To date, four nomogram studies regarding SCLC have been published, ${ }^{13-16}$ however all the studies include allstaged SCLC patients and did not analyze patients who underwent surgery. Thus, we aimed to establish a nomogram to predict survival outcomes after surgery in limitedstage SCLC using a large cohort from the Surveillance, Epidemiology, and End Results (SEER) database. In addition, this nomogram model was externally validated by a separate cohort from the Cancer Institute and Hospital of the Chinese Academy of Medical Sciences (CICAMS).

\section{PATIENTS AND METHODS}

\section{Study Population}

The SEER is a population-based database that covers approximately $28 \%$ of the US population. The latest SEER data, released in April 2019, includes cancer incidence data ranging from January 1975 to December 2016. A total of 94,247 SCLC cases were identified from the database using SEER* Stat version 8.3.5 (National Cancer Institute, Bethesda, MD, USA), of which 1006 resected limited-stage cases met our inclusion criteria and entered the training cohort. The specific criteria and codes for inclusion or exclusion are shown in electronic supplementary Fig. S1.

To examine the generalizability of this model, an external validation cohort was constructed from the CICAMS. We reviewed our database of patients with histologically confirmed SCLC from January 2000 to December 2015. A total of 444 consecutive resected limited-stage SCLC patients were identified. Laboratory tests, pulmonary function test, computed tomography of the chest and upper abdomen, bronchoscopy, brain magnetic resonance imaging, emission computed tomography bone scans, or positron emission tomography of the whole body were routinely performed prior to surgery at our institution. Clinical data were retrieved from the medical record database and survival information was obtained from our follow-up center or by contacting the patients. Ethical approval was given by the Research Ethics Committee of CICAMS, which waived the requirement for informed patient consent because of the retrospective nature of this study.

\section{Variables}

For each patient, several variables were gathered from the SEER database, i.e. age, sex, race, tumor location, surgery, number of lymph nodes dissected (LND), number of lymph node metastases (LNM), histology type, stage, additional treatment (chemotherapy or radiotherapy), survival months, causes of death, and vital status. For the validation cohort, the same variables were also extracted. In terms of surgery, surgical codes indicating the resection of fewer than one lobe (wedge resection, segmental resection) were categorized as sublobectomy. The combined histology subtype refers to SCLC accompanied by other components (such as adenocarcinoma or squamous carcinoma). In addition, we revised the TNM categories according to the Collaborative Staging Manual and Coding Instructions for the AJCC 8th staging system. ${ }^{17} \mathrm{We}$ assembled the IA1, IA2, IA3 stages as IA disease as no significant difference in survival was found among these substages. $^{18}$ According to the SEER summary staging system, we further divided VALSG limited disease into two subgroups: localized disease (tumor confined to the primary organ without LNM) and regional disease (tumor invaded directly to the adjacent organ/tissue or regional LNM). Information regarding chemotherapy or radiotherapy was also included, as additional therapy; however, we were unable to define neoadjuvant or adjuvant therapy due to the lack of sequence of the additional treatment. In the SEER database, regretfully, variable of visceral pleural invasion for lung cancer was unavailable before 2010, information regarding prophylactic cranial radiation was missing during this period, and more than one-third of the differentiation grade were undefined, hence we did not include these parameters in the analysis.

\section{Construction of the Nomogram}

The nomogram was developed using a training cohort of 1006 patients. Variables entered into the final analysis included age, sex, race, laterality, primary site, surgery, LND, LNM, histology type, TNM stage, chemotherapy, and radiotherapy. Overall survival (OS) was calculated according to vital status, and censored subjects were 
recorded based on the status of 'alive', for OS. Significant prognostic correlating variables were analyzed using the univariate Cox proportional hazards regression model and the Wald test. Variables with a $p$ value $<0.05$ entered the multivariate Cox regression analysis for eliminating redundant variables via the backward stepwise process based on Akaike information criterion. ${ }^{19}$ The prognostic nomogram was constructed based on the risk score calculated by the final Cox regression model.

\section{Model Performance and Validation}

The performance for predicting survival of this nomogram model was evaluated using the concordance index (C-index), which represents a concordance measure analogous to the area under the receiver operating characteristic (ROC). The C-index ranges from 0.5 (indicating no better than random chance) to 1.0 (indicating perfect prediction). ${ }^{20}$ Calibration curves of the nomogram for 1-, 3-, and 5-year survival were plotted to evaluate the consistency between predicted survival probability and actual survival proportion. A perfectly calibrated model would present with a 45-degree curve. For model validation, 1000 bootstrap resamples in the training cohort were applied for internal validation. Furthermore, an independent external validation was conducted using the CICAMS cohort. The two conventional staging models-AJCC 8th TNM staging system and VALSG staging system-were also assessed for the prognostic performance, in both the training and validation cohorts. For the present study, the VALSG system incorporated modified localized disease and regional disease. In addition, the area under the curve (AUC) of the time-dependent ROC was calculated each month, from months 1 to 60. The decision curve analysis (DCA) was also conducted to evaluate the benefits and advantages of our new predicting model over the other two staging models.

For assessing the discriminate ability of the model, we also grouped patients into several risk subsets according to prognostic scores in the training cohort. The cut-off values were defined using the X-tile software version 3.6.1 (Yale University School of Medicine, New Haven, CT, USA), which could recognize the optimal cut-off values for continuous variables through calculating the largest Chi square and minimum $p$ values. These cut-off values were then applied to the different TNM categories and the validation cohort; the respective log-rank $p$ values were calculated to compare the difference in survival.

\section{Statistical Analysis}

Statistical analysis was performed using SPSS 23.0 (IBM Corporation, Armonk, NY, USA) and R version 3.6.1
(The R Foundation for Statistical Computing, Vienna, Austria). The R packages 'survival' (version 2.44-1.1), 'foreign' (version 0.8-72), 'rms' (version 5.1-3.1), 'survivminer' (version 0.4.5), and 'timeROC' (version 1.0.3) were used for nomogram construction and evaluation. Furthermore, the R packages 'DynNom' (version 5.0.1) and 'rsconnect' (version 0.8.16) were applied for developing a user-friendly web-based interface for our nomogram. Kaplan-Meier survival analysis was used to assess distinctions in prognosis with a log-rank $p$ value. A two-tailed $p$ value $<0.05$ was considered to be statistically significant.

\section{RESULTS}

\section{Characteristics of the Training and Validation Cohorts}

The training cohort comprised 1006 patients with resected primary limited-stage SCLC, from the SEER database. There were 755 deaths over a median follow-up duration of 101.0 months (range 1-203 months). The validation cohort consisted of 444 cases of limited-stage SCLC and 222 deaths were observed over a median followup duration of 80.0 months (range 2 days to 213 months). Detailed demographic characteristics of patients in the training and validation cohorts are shown in Table 1. The median age at diagnosis was 63.5 years (range 34-90 years) and 50.5 years (range 19-82 years) in the training and validation cohorts, respectively. Both groups were predominant in the male sex. Lobectomy accounted for the major procedure in all enrolled cases. Over $10 \%$ of cases were diagnosed with combined SCLC in both groups.

\section{Independent Prognostic Factors in the Training Cohort}

Cox proportional hazards models were performed to assess the independent prognostic factors in the training cohort and the results are shown in Table 2. In univariate analysis, age, sex, surgery, $T$ stage, $N$ stage, LND, LNM, and chemotherapy were revealed to be significant correlating variables for OS. The univariate analysis survival curves are shown in electronic supplementary Fig. S2. N stage was not an independent variable for LNM and was hence excluded from the multivariate analysis. After multivariate analysis, age, sex, surgery, T stage, LND, LNM, and chemotherapy were demonstrated to be independent prognostic factors.

\section{Developing the Prognostic Nomogram Model}

Significant variables of age, sex, surgery, T stage, LND, LNM, and chemotherapy were finally selected for the 
TABLE 1 Demographic characteristics of the training and validation cohorts

\begin{tabular}{|c|c|c|c|}
\hline Demographic characteristics & Overall & Training cohort & Validation cohort \\
\hline No. of patients & 1450 & 1006 & 444 \\
\hline \multicolumn{4}{|l|}{ Age, years } \\
\hline$<60$ & $508(35.0)$ & $261(25.9)$ & $247(55.6)$ \\
\hline $60-70$ & $542(37.4)$ & 399 (39.7) & $143(32.2)$ \\
\hline$>70$ & $400(27.6)$ & $346(34.4)$ & $54(12.2)$ \\
\hline \multicolumn{4}{|l|}{ Sex } \\
\hline Female & $647(44.6)$ & $534(53.1)$ & $113(25.5)$ \\
\hline Male & $803(55.4)$ & $472(46.9)$ & $331(74.5)$ \\
\hline \multicolumn{4}{|l|}{ Race } \\
\hline White & $910(62.8)$ & $910(90.5)$ & - \\
\hline Black & $73(5.0)$ & $73(7.3)$ & - \\
\hline Other & $23(1.6)$ & $23(2.3)$ & - \\
\hline \multicolumn{4}{|l|}{ Laterality } \\
\hline Left & $668(46.1)$ & $451(44.8)$ & $217(48.9)$ \\
\hline Right & $782(53.9)$ & $555(55.2)$ & $227(51.1)$ \\
\hline \multicolumn{4}{|l|}{ Primary site } \\
\hline Upper lobe & $773(53.3)$ & $582(57.9)$ & $191(43.0)$ \\
\hline Middle lobe & $87(6.0)$ & $60(6.0)$ & $27(6.1)$ \\
\hline Lower lobe & $533(36.8)$ & $307(30.5)$ & $226(50.9)$ \\
\hline Unknown & $57(3.9)$ & $57(5.7)$ & $0(0.0)$ \\
\hline \multicolumn{4}{|l|}{ Surgery } \\
\hline Lobectomy & $1022(70.5)$ & $622(61.8)$ & $400(90.1)$ \\
\hline Sublobectomy & $356(24.6)$ & 347 (34.5) & $9(2.0)$ \\
\hline Pneumonectomy & $72(5.0)$ & $37(3.7)$ & $35(7.9)$ \\
\hline \multicolumn{4}{|l|}{ Histology } \\
\hline Pure SCLC & 1231 (84.9) & $834(82.9)$ & $397(89.4)$ \\
\hline Combined SCLC & $219(15.1)$ & $172(17.1)$ & 47 (10.6) \\
\hline \multicolumn{4}{|l|}{ LND group } \\
\hline $0-5$ & $546(37.7)$ & $531(52.8)$ & $15(3.4)$ \\
\hline $6-10$ & $282(19.4)$ & $238(23.7)$ & 44 (9.9) \\
\hline $11-20$ & 346 (23.9) & $171(17.0)$ & $175(39.4)$ \\
\hline $21-30$ & 170 (11.7) & $44(4.4)$ & $126(28.4)$ \\
\hline$>30$ & $106(7.3)$ & $22(2.2)$ & $84(18.9)$ \\
\hline \multicolumn{4}{|l|}{ LNM group } \\
\hline 0 & $637(43.9)$ & $460(45.7)$ & 177 (39.9) \\
\hline $1-3$ & $418(28.8)$ & $279(27.7)$ & $139(31.3)$ \\
\hline $4-6$ & $107(7.4)$ & $44(4.4)$ & $63(14.2)$ \\
\hline $7-9$ & $48(3.3)$ & $21(2.1)$ & $27(6.1)$ \\
\hline$\geq 10$ & $42(2.9)$ & $8(0.8)$ & $34(7.7)$ \\
\hline NO* & 198 (13.7) & $194(19.3)$ & $4(0.9)$ \\
\hline \multicolumn{4}{|l|}{ AJCC stage } \\
\hline IA & $377(26.0)$ & $303(30.1)$ & $74(16.7)$ \\
\hline IB & $204(14.1)$ & $142(14.1)$ & $62(14.0)$ \\
\hline IIA & $63(4.3)$ & 39 (3.9) & $24(5.4)$ \\
\hline IIB & $325(22.4)$ & $211(21.0)$ & $114(25.7)$ \\
\hline IIIA & 375 (25.9) & 237 (23.6) & $138(31.1)$ \\
\hline IIIB & $106(7.3)$ & $74(7.4)$ & $32(7.2)$ \\
\hline \multicolumn{4}{|l|}{$T$ stage } \\
\hline$T 1 \mathrm{a}$ & $98(6.8)$ & $76(7.6)$ & $22(5.0)$ \\
\hline$T 1 \mathrm{~b}$ & $284(19.6)$ & $226(22.5)$ & $58(13.1)$ \\
\hline
\end{tabular}


TABLE 1 (continued)

\begin{tabular}{llll}
\hline Demographic characteristics & Overall & Training cohort & Validation cohort \\
\hline$T 1 \mathrm{c}$ & $228(15.7)$ & $159(15.8)$ & $69(15.5)$ \\
$T 2 \mathrm{a}$ & $402(27.7)$ & $265(26.3)$ & $137(30.9)$ \\
$T 2 \mathrm{~b}$ & $140(9.7)$ & $65(6.5)$ & $75(16.9)$ \\
$T 3$ & $174(12.0)$ & $112(11.1)$ & $62(14.0)$ \\
$T 4$ & $124(8.6)$ & $103(10.2)$ & $21(4.7)$ \\
$N$ stage & & & $181(40.8)$ \\
$N 0$ & $761(52.5)$ & $580(57.7)$ & $128(28.8)$ \\
$N 1$ & $332(22.9)$ & $204(20.3)$ & $135(30.4)$ \\
$N 2$ & $357(24.6)$ & $222(22.1)$ & $170(38.3)$ \\
VALSG stage & & & $274(61.7)$ \\
Localized & $658(45.4)$ & $488(48.5)$ & $34(7.7)$ \\
Regional & $792(54.6)$ & $518(51.5)$ & $410(92.3)$ \\
Chemotherapy & & $333(33.1)$ & $324(73.0)$ \\
No/unknown & $367(25.3)$ & $673(66.9)$ & $120(27.0)$ \\
Yes & $1083(74.7)$ & $608(60.4)$ & \\
Radiotherapy & & $398(39.6)$ & \\
No/unknown & $932(64.3)$ & & \\
Yes & $518(35.7)$ & & \\
\hline
\end{tabular}

Data are expressed as $n(\%)$

$S C L C$ small cell lung cancer, $L N D$ lymph node dissected, $L N M$ lymph node metastasis, $N O^{*}$ no lymph nodes dissected, AJCC American Joint Committee on Cancer, VALSG Veterans Administration Lung Study Group

development of the nomogram model. Each variable was assigned to a point score ranging from 0 to 10 (electronic supplementary Table S1). In the nomogram for OS, LNM showed the largest contribution to prognosis, with a point score of 10 , followed by age and T stage (Fig. 1). Notably, sublobectomy and pneumonectomy demonstrated an approximately equal contribution for survival prediction. The individual risk scores were calculated by summing up the score of each variable, and the probabilities of survival at 1,3 , and 5 years were easily determined by locating its corresponding point on the survival scale.

\section{Model Performance and Validation of the Nomogram}

In the training cohort, the $\mathrm{C}$-indexes for the established nomogram were $0.723(95 \%$ confidence interval [CI] $0.685-0.761), 0.722$ (95\% CI 0.690-0.755), and 0.746 (95\% CI 0.710-0.781) for 1-, 3-, and 5-year OS, respectively, and 0.816 (95\% CI 0.762-0.870), $0.710(95 \% \mathrm{CI}$ $0.658-0.760)$, and 0.693 (95\% CI 0.637-0.750), respectively, in the validation cohort. The calibration plots at 1-, 3-, and 5-year survival showed excellent consistency in the training cohort and acceptable consistency in the validation cohort between the predicted survival probability and actual observation (Fig. 2).
With regard to prognostic ability, we also conducted comparisons of the model performance between our nomogram and the two conventional staging systems. The 1-, 3-, and 5-year time-dependent ROC curves of the three models are shown in Fig. 3. In the training cohort, all AUCs of the nomogram model were significantly higher than the AJCC $(p<0.0001)$ or VALSG $(p<0.0001)$ staging systems. Similar results were also observed in the validation cohort for comparing this nomogram model with the AJCC or VALSG staging systems, which verified the strong and robust prognostic power of this nomogram. DCA analysis showed that our nomogram model provided significantly increased net benefits over the AJCC or VALSG staging systems within wide and practical ranges of threshold probabilities (Fig. 3), which further verified the better prognostic performance of our nomogram in clinical appliance. Furthermore, we compared the continuous trends of the prognostic performance of each model, and found the AUCs of our nomogram model were higher than that of the AJCC and VALSG staging systems throughout the calculation period (from months 1 to 60), whether in the training or validation cohorts (Fig. 4). 
TABLE 2 Results of univariable and multivariate Cox proportional hazards regression analysis for overall survival

\begin{tabular}{|c|c|c|c|c|}
\hline \multirow[t]{2}{*}{ Variables } & \multicolumn{2}{|l|}{ Univariable analysis } & \multicolumn{2}{|l|}{ Multivariate analysis } \\
\hline & HR $(95 \%$ CI $)$ & $p$ Value & $\operatorname{HR}(95 \% \mathrm{CI})$ & $p$ Value \\
\hline \multicolumn{5}{|l|}{ Age, years } \\
\hline$<60$ & 1 & & 1 & \\
\hline $60-70$ & $1.369(1.133-1.654)$ & 0.001 & $1.545(1.273-1.876)$ & $<0.001$ \\
\hline$>70$ & $1.918(1.585-2.322)$ & $<0.001$ & $2.057(1.686-2.510)$ & $<0.001$ \\
\hline \multicolumn{5}{|l|}{ Sex } \\
\hline Female & 1 & & 1 & \\
\hline Male & $1.302(1.128-1.502)$ & $<0.001$ & $1.324(1.145-1.532)$ & $<0.001$ \\
\hline \multicolumn{5}{|l|}{ Race } \\
\hline White & 1 & & & \\
\hline Black & $0.770(0.576-1.029)$ & 0.077 & & \\
\hline Other & $0.819(0.506-1.325)$ & 0.415 & & \\
\hline \multicolumn{5}{|l|}{ Laterality } \\
\hline Left & 1 & & & \\
\hline Right & $1.021(0.885-1.179)$ & 0.772 & & \\
\hline \multicolumn{5}{|l|}{ Primary site } \\
\hline Upper lobe & 1 & & & \\
\hline Middle lobe & $0.939(0.687-1.285)$ & 0.695 & & \\
\hline Lower lobe & $1.059(0.902-1.243)$ & 0.482 & & \\
\hline Unknown & $1.338(0.993-1.803)$ & 0.056 & & \\
\hline \multicolumn{5}{|l|}{ Surgery } \\
\hline Lobectomy & 1 & & 1 & \\
\hline Sublobectomy & $1.506(1.297-1.749)$ & $<0.001$ & $1.313(1.092-1.579)$ & 0.004 \\
\hline Pneumonectomy & $1.444(0.997-2.091)$ & 0.052 & $1.294(0.882-1.898)$ & 0.187 \\
\hline \multicolumn{5}{|l|}{ Histology } \\
\hline Pure SCLC & 1 & & & \\
\hline Combined SCLC & $0.987(0.815-1.195)$ & 0.890 & & \\
\hline \multicolumn{5}{|l|}{$T$ stage } \\
\hline$T 1 \mathrm{a}$ & 1 & & 1 & \\
\hline$T 1 \mathrm{~b}$ & $1.282(0.925-1.775)$ & 0.136 & $1.263(0.909-1.754)$ & 0.165 \\
\hline$T 1 \mathrm{c}$ & $1.382(0.985-1.941)$ & 0.062 & $1.341(0.951-1.892)$ & 0.094 \\
\hline$T 2 \mathrm{a}$ & $1.560(1.136-2.143)$ & 0.006 & $1.621(1.174-2.238)$ & 0.003 \\
\hline$T 2 \mathrm{~b}$ & $1.427(0.956-2.130)$ & 0.082 & $1.737(1.146-2.635)$ & 0.009 \\
\hline$T 3$ & $2.020(1.423-2.868)$ & $<0.001$ & $1.928(1.345-2.764)$ & $<0.001$ \\
\hline$T 4$ & $2.301(1.614-3.281)$ & $<0.001$ & $2.051(1.432-2.938)$ & $<0.001$ \\
\hline \multicolumn{5}{|l|}{$N$ stage } \\
\hline NO & 1 & & NA & NA \\
\hline$N 1$ & $1.525(1.275-1.825)$ & $<0.001$ & & \\
\hline$N 2$ & $1.852(1.555-2.206)$ & $<0.001$ & & \\
\hline \multicolumn{5}{|l|}{ LND } \\
\hline $0-5$ & 1 & & 1 & \\
\hline $6-10$ & $0.754(0.631-0.901)$ & 0.002 & $0.838(0.681-1.032)$ & 0.096 \\
\hline $11-20$ & $0.709(0.577-0.871)$ & 0.001 & $0.691(0.543-0.879)$ & 0.003 \\
\hline $21-30$ & $0.759(0.527-1.092)$ & 0.137 & $0.650(0.437-0.967)$ & 0.034 \\
\hline$>30$ & $0.542(0.318-0.926)$ & 0.025 & $0.525(0.301-0.915)$ & 0.023 \\
\hline \multicolumn{5}{|l|}{ LNM } \\
\hline 0 & 1 & & 1 & \\
\hline $1-3$ & $1.800(1.515-2.138)$ & $<0.001$ & $1.813(1.510-2.176)$ & $<0.001$ \\
\hline $4-6$ & $2.200(1.567-3.089)$ & $<0.001$ & $2.594(1.822-3.695)$ & $<0.001$ \\
\hline
\end{tabular}


TABLE 2 (continued)

\begin{tabular}{|c|c|c|c|c|}
\hline \multirow[t]{2}{*}{ Variables } & \multicolumn{2}{|l|}{ Univariable analysis } & \multicolumn{2}{|l|}{ Multivariate analysis } \\
\hline & HR $(95 \%$ CI $)$ & $p$ Value & HR $(95 \%$ CI $)$ & $p$ Value \\
\hline $7-9$ & $2.280(1.415-3.672)$ & 0.001 & $3.297(1.995-5.448)$ & $<0.001$ \\
\hline$\geq 10$ & $4.378(2.163-8.864)$ & $<0.001$ & $7.065(3.38-14.767)$ & $<0.001$ \\
\hline NO* & $1.844(1.521-2.236)$ & $<0.001$ & $1.458(1.162-1.830)$ & 0.001 \\
\hline \multicolumn{5}{|l|}{ Chemotherapy } \\
\hline No/unknown & 1 & & 1 & \\
\hline Yes & $0.842(0.724-0.979)$ & 0.025 & $0.721(0.613-0.848)$ & $<0.001$ \\
\hline \multicolumn{5}{|l|}{ Radiotherapy } \\
\hline No/unknown & 1 & & & \\
\hline Yes & $1.036(0.896-1.198)$ & 0.632 & & \\
\hline
\end{tabular}

$H R$ hazard ratio, $C I$ confidence interval, $S C L C$ small cell lung cancer, $L N D$ lymph node dissected, $L N M$ lymph node metastasis, $N O^{*}$, no lymph nodes dissected, $N A$ not included in the model due to interference with LNM

FIG. 1 Nomograms for predicting postoperative overall survival in resected limitedstage SCLC patients. $L N D$ lymph node dissected, $L N M$ lymph node metastasis, $N O^{*}$ no lymph nodes dissected, $S C L C$ small cell lung cancer

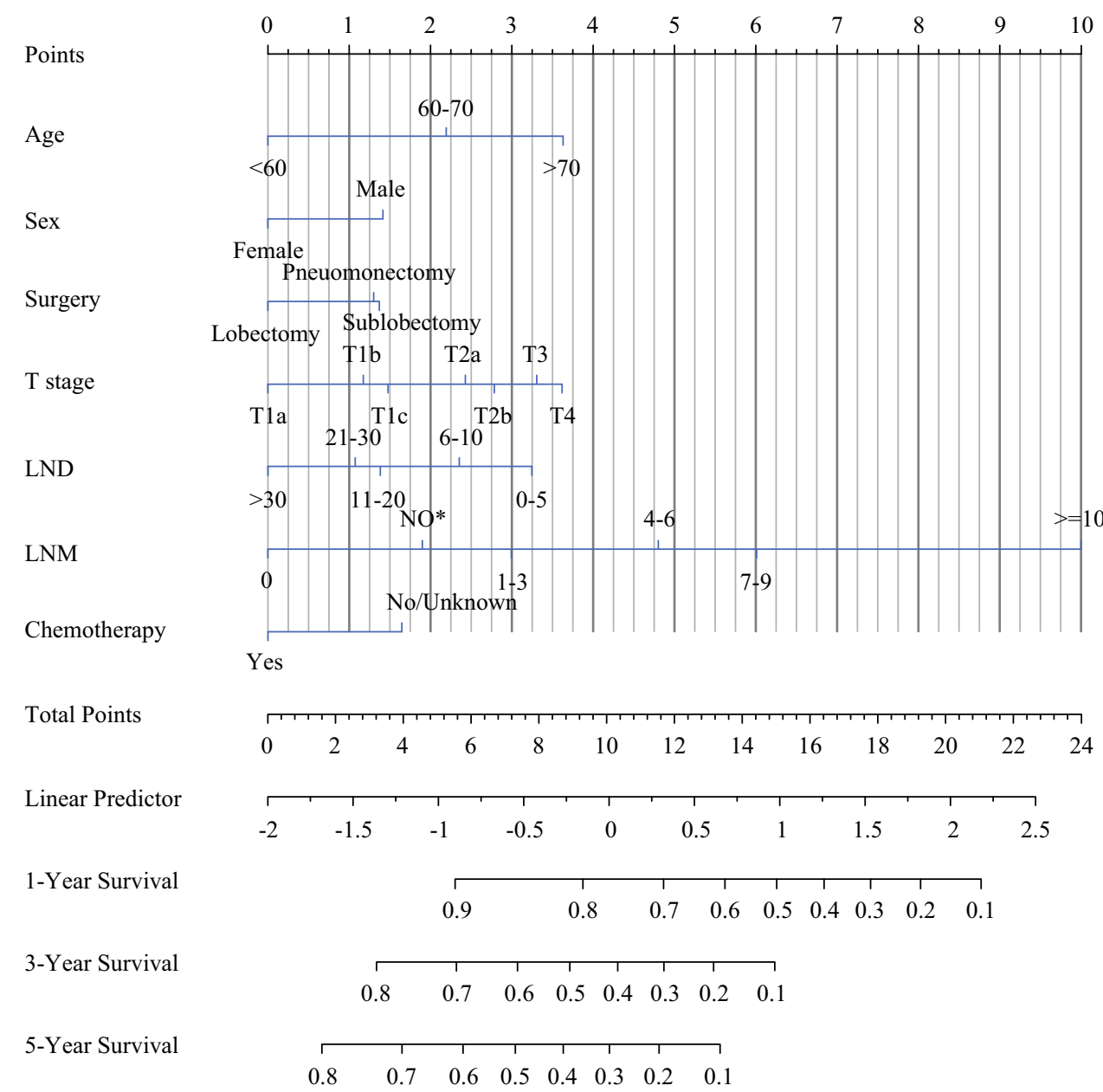




\section{(a)}

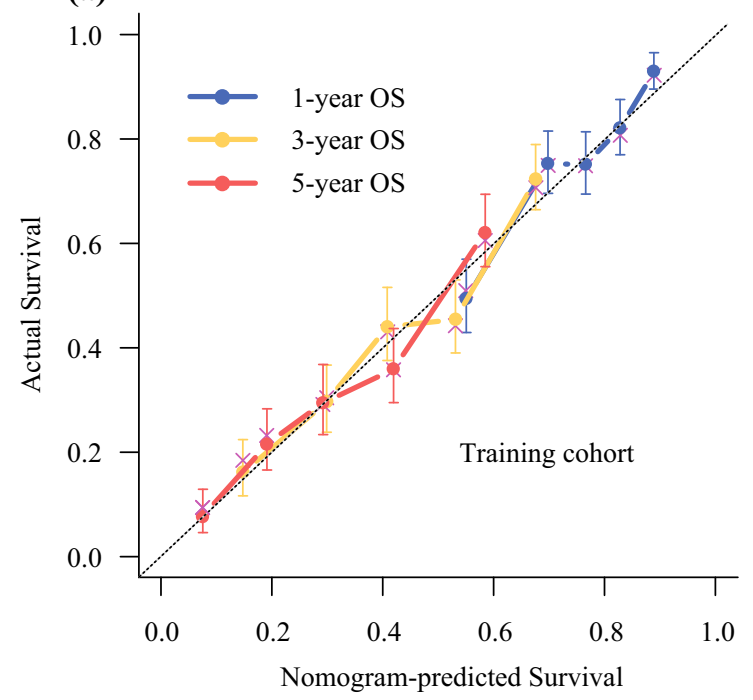

FIG. 2 Calibration plots for nomogram-predicted survival ( $x$-axis) and actual observed survival ( $y$-axis). Calibration curves for OS in the a training and b validation cohorts; curves for 1-, 3-, and 5-year OS

\section{Risk-Stratifying Ability of the Nomogram}

Based on the total predictive risk scores, we subcategorized the training cohort into four risk groups, with the optimal cut-off values developed from X-tile software. Detailed subgroups were 0-7.96, 7.97-10.13, 10.14-12.05, and 12.06-20.31 (electronic supplementary Fig. S3). The survival curves for OS showed significant distinctions between any two adjacent groups $(p<0.0001)$ in the training cohort (Fig. 5a) Significant differences were also observed between subgroups when patients were stratified by AJCC stages $(p<0.0001)$ (Fig. $5 b-d)$. This grouping method was then applied to the validation cohort and significant distinctions in survival between different risk groups were also observed, even within certain AJCC staging categories (Fig. 5e-h).

\section{Webserver Development for the Nomogram}

For convenient application of our nomogram, we developed dynamic calculators (electronic supplementary Fig. S4) on the basis of a user-friendly website (https://ze ngqp1991.shinyapps.io/zengmodel/), which could be used directly by researchers and clinicians. By inputting certain clinical variables, we can easily obtain the corresponding individualized predicted survival probabilities through the output data generated by the website.

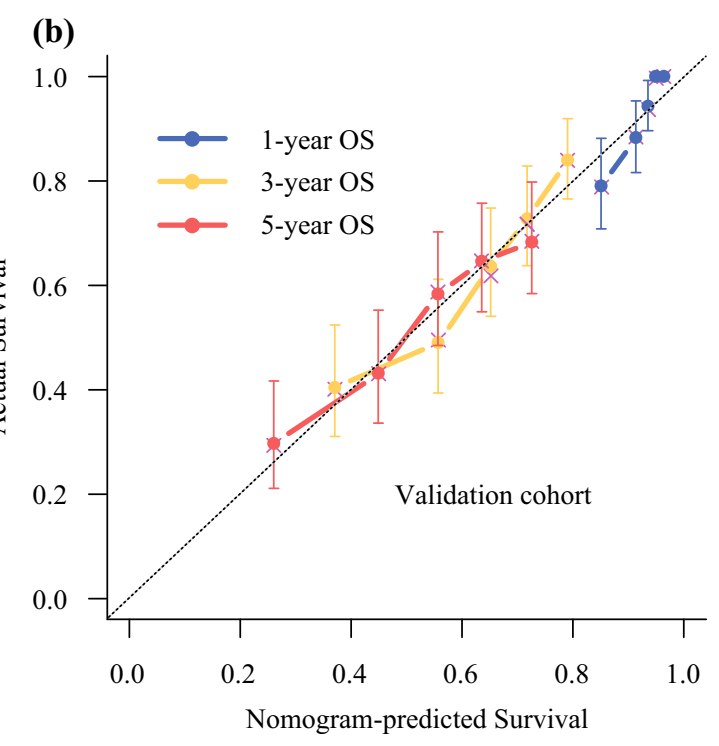

were presented as blue, yellow, and red lines, respectively. Error bars represent $95 \%$ confidence intervals. OS overall survival

\section{DISCUSSION}

Since surgical resection remains an indispensable treatment for early-stage SCLC, and because of the impreciseness of the commonly used AJCC or VALSG staging system for predicting survival for SCLC, a welldeveloped prognostic model was warranted to compensate for these limitations. In the present study, a novel nomogram prognostic model was established from a large population-based database of limited-stage resected SCLC, and validated using a cohort from our institution. Based on the common clinicopathological variables and treatment information, the individualized probability of survival is readily obtained through our easily accessible online calculator, which could help clinicians in treatment decision making or design of clinical trials. To our knowledge, this was the first attempt to establish a prediction model for the long-term survival of resectable, limited-stage SCLC patients.

Several previous studies have published nomograms regarding survival prediction for SCLC. In 2015, Xie and colleagues developed a nomogram, from a cohort of 938 cases, for predicting OS for SCLC, incorporating peripheral blood markers, ${ }^{13}$ while in 2017, Xiao et al. demonstrated a prognostic nomogram for SCLC patients using a single-institutional cohort of 647 cases. ${ }^{15}$ Regretfully, neither of the two studies applied the more accurate TNM staging system, nor did they assign an independent validation for the model. Recently, Wang et al. developed and validated a web-accessible nomogram for predicting the survival of SCLC patients using the National Cancer 
(a)

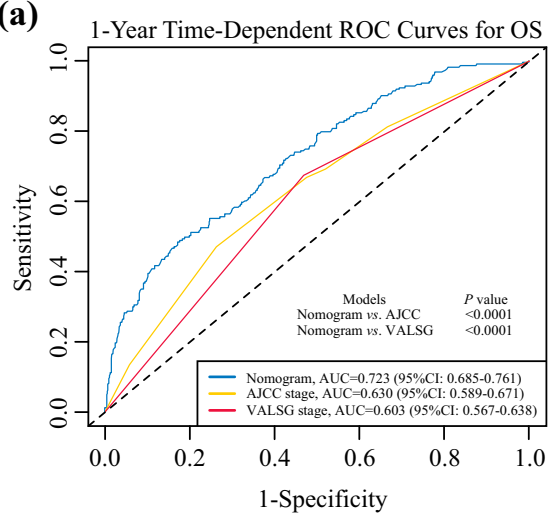

(d)

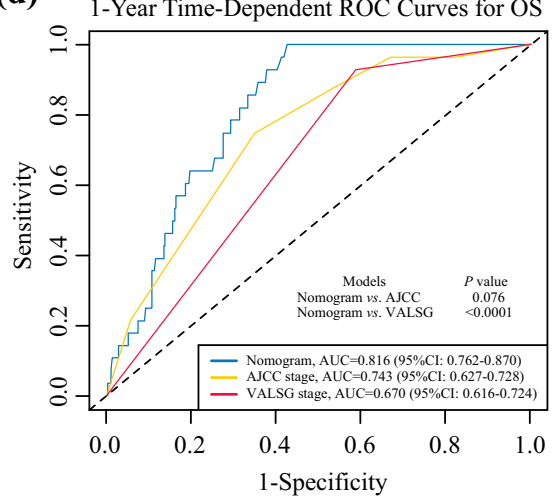

(g)

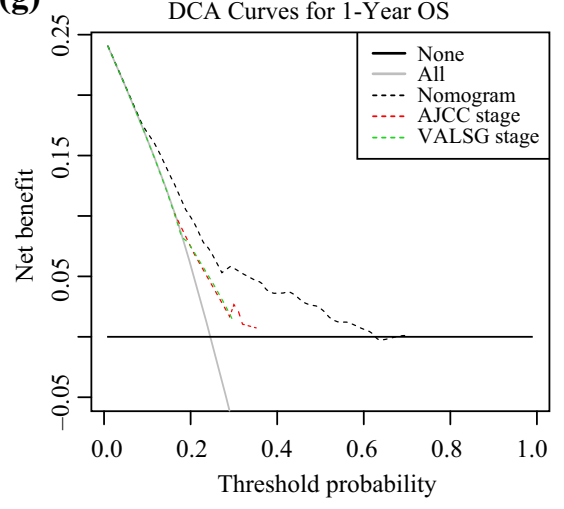

(b)

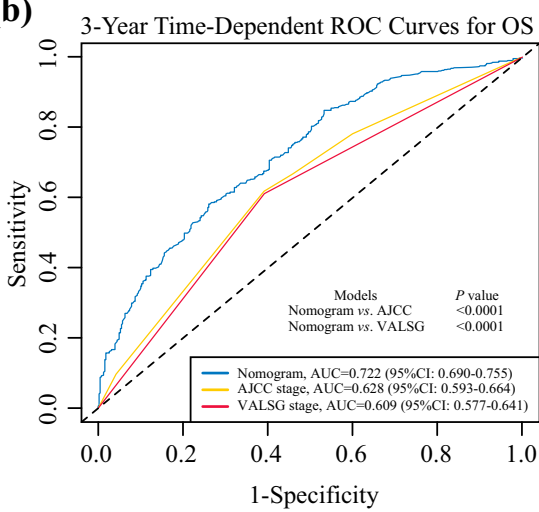

(e)

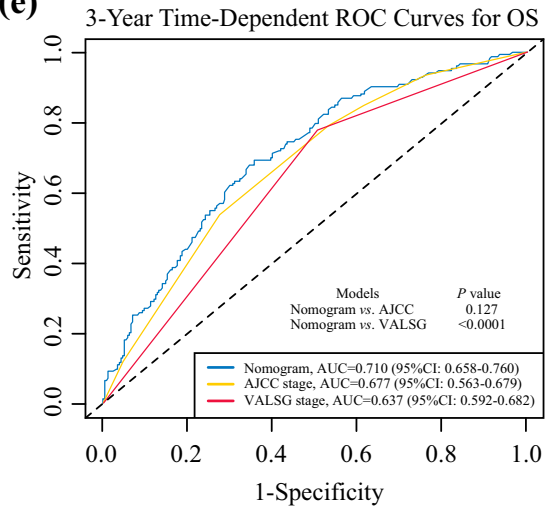

(h)

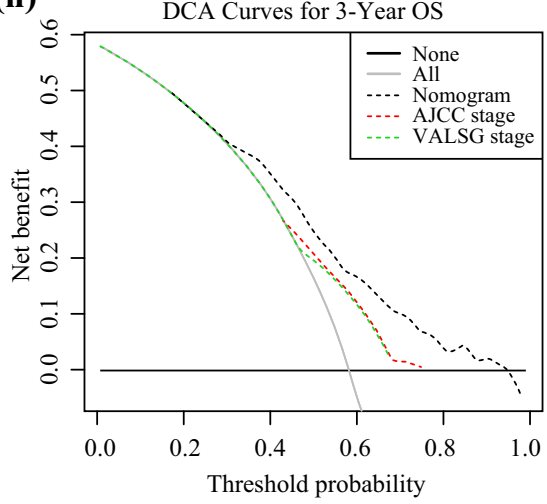

(c)

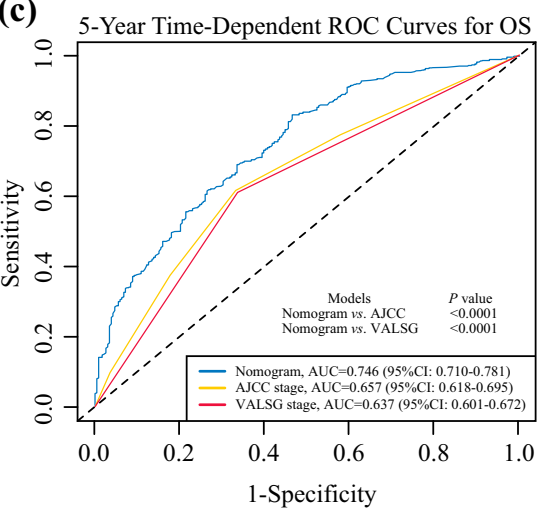

(f)

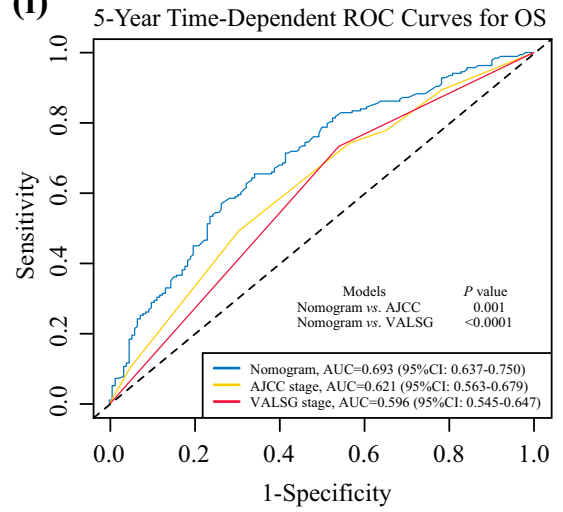

(i)

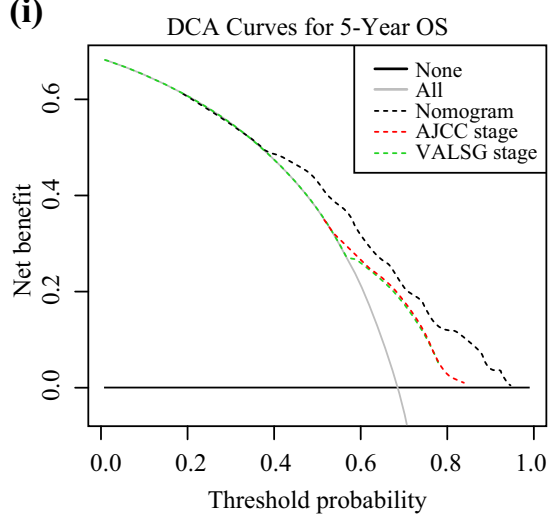

FIG. 3 Model performance of the proposed nomogram. a-f Timedependent ROC curves of the three prognostic models for predicting 1-, 3-, and 5-year OS. The AUCs of the three prognostic models at each time point of interest were presented and compared in the training and validation cohorts. (g-i) DCA curves of the three prognostic models for 1-, 3-, and 5-year OS. The $x$-axis represents the

Database (NCDB). ${ }^{16}$ Despite the large sample size, this model incorporated the entire stages and treatment patterns, including surgery, chemotherapy, and radiotherapy, which failed to eliminate bias from the interactions between stages and treatment strategies. In our nomogram, we established a surgically based prognostic model and included the limited-stage SCLC cases, which could provide a more accurate probability of survival for this specific subset of patients. The training cohort was obtained from threshold probabilities and the y-axis measures the net benefit. $R O C$ receiver operating characteristic, $A U C$ area under the curve, $D C A$ decision curve analysis, $O S$ overall survival, AJCC American Joint Committee on Cancer, VALSG Veterans Administration Lung Study Group, $C I$ confidence interval

the large and wide geographically distributed SEER database, which guaranteed its generalizability for SCLC patients. Furthermore, this nomogram was validated in an independent cohort of Chinese patients, which increased the universality of this nomogram.

Through univariate and multivariate analysis, age, sex, surgery, $T$ stage, LND, LNM, and chemotherapy were recognized as independent prognostic parameters, which was in high accordance with previous reports. ${ }^{11,16,21-23}$ 

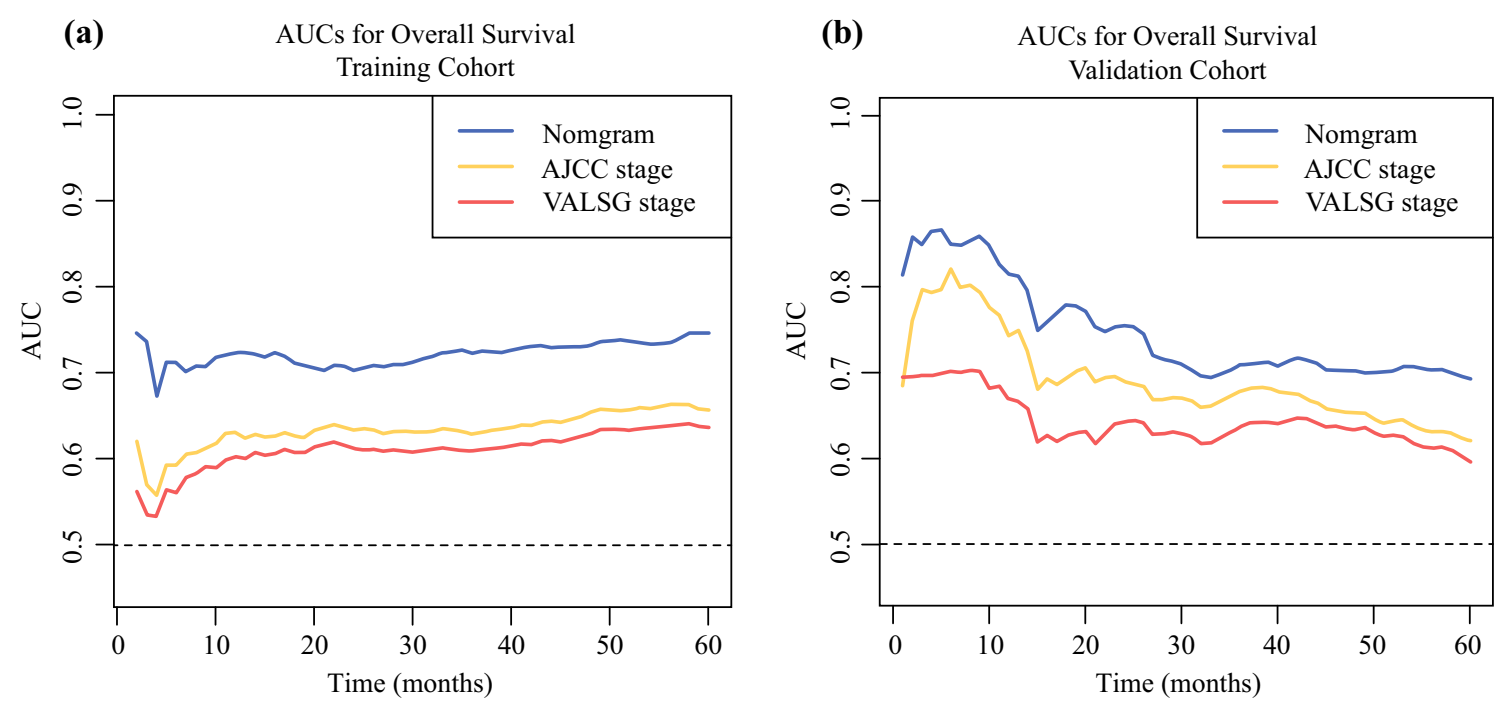

FIG. 4 Continuous AUCs of the three prognostic models in the a training and $\mathbf{b}$ validation cohorts throughout the time period of 1-60 months. $A U C$ area under the curve, AJCC American Joint Committee on Cancer, VALSG Veterans Administration Lung Study Group
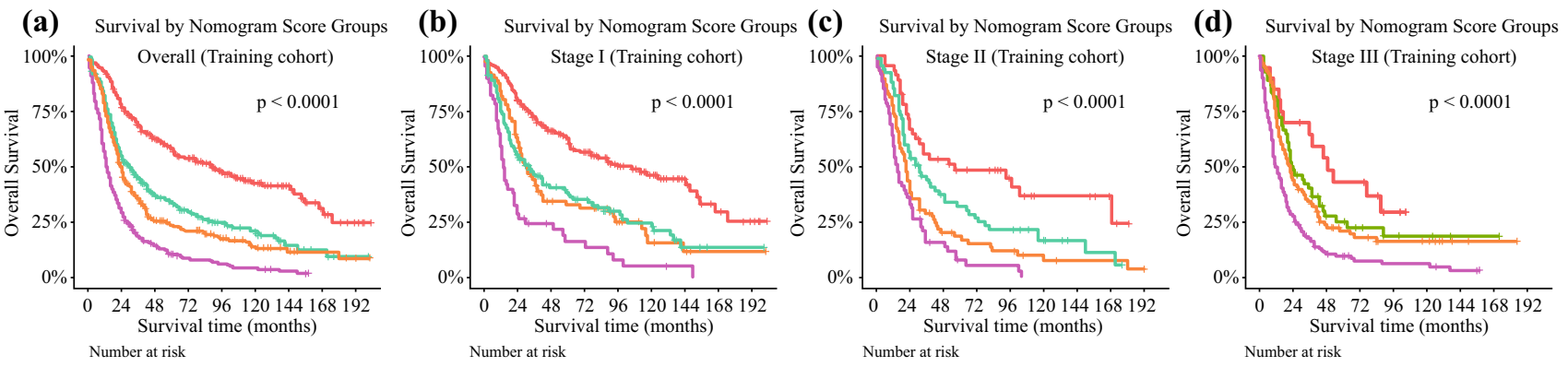

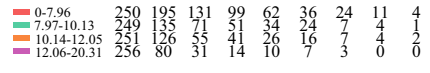

(e) Survival by Nomogram Score Groups

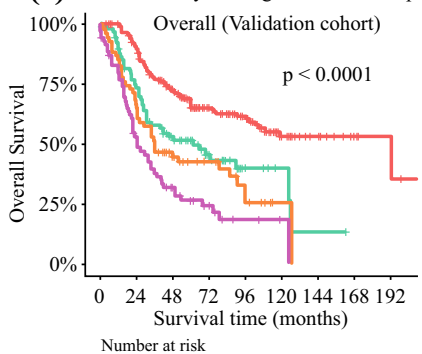

Number at risk Number at risk

Number at risk
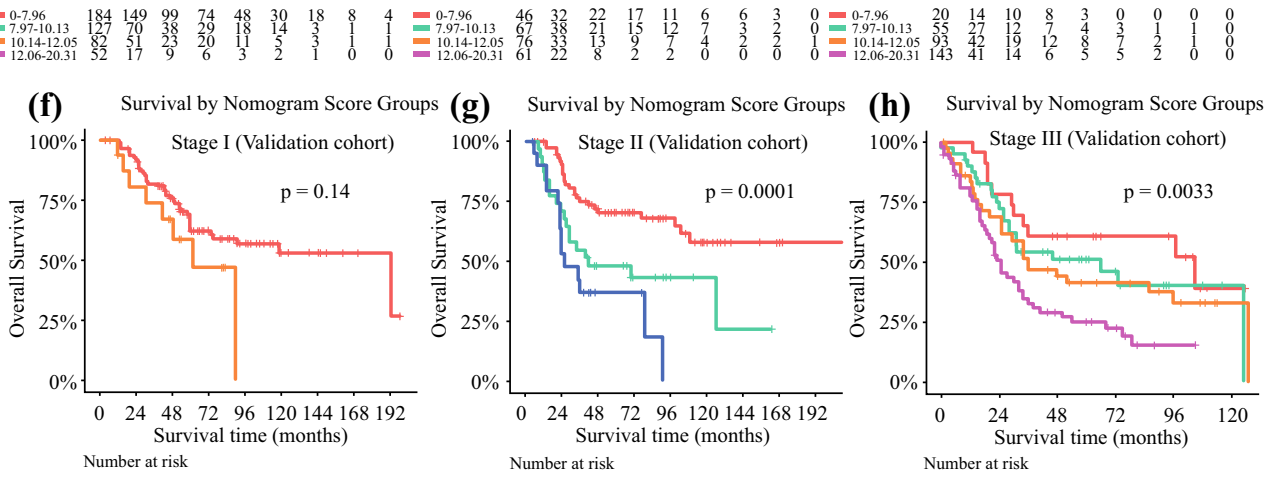

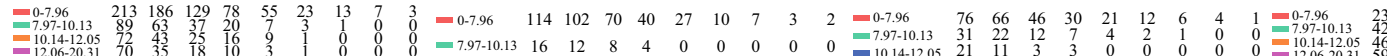

FIG. 5 Determinations of risk score groups based on the training cohort and the corresponding survival curves for overall survival in the overall and stage-stratified patients in the $\mathbf{a}-\mathbf{d}$ training and $\mathbf{e}-\mathbf{h}$ validation cohorts. Subgroups with fewer than 10 patients were omitted from the graphs

Notably, radiotherapy was revealed not to be a significant prognostic factor in our study, which may be attributed to the contradictory impact of radiotherapy on patients with different $N$ statuses. Wong et al. ${ }^{12}$ reported that radiotherapy deteriorated OS in NO SCLC patients, but improved survival in N2-stage patients. As shown in electronic supplementary Table S2, similar effects were also observed in our study. Therefore, to avoid assigning incorrect risk scores to the unspecified patients and to maintain the convenience of this model for clinical use, we did not conduct a subanalysis for prognostic models incorporating radiotherapy. In addition, histology type was not significantly correlated with survival in the univariate analysis, which was contradictory to other studies. In their study, Zhao et al. ${ }^{24}$ reported that combined SCLC was associated with decreased OS compared with pure SCLC. 
Yokouchi et al. ${ }^{25}$ conducted a retrospective multicenter analysis of 156 resected SCLC patients and revealed no impact of histology type on survival. Given the inconsistency of these studies, we hence excluded histology type from the nomogram construction.

To avoid the overfitting of this nomogram, it was necessary to apply model validation and calibration. In our study, the calibration curve showed optimal accordance between predicted survival probability and actual observation, which revealed good repeatability and reliability of this established model. Furthermore, this nomogram model fits well in the external Chinese validation cohort, which supported the universalized application of this nomogram despite ethnic and geographical differences.

Although the C-indexes of our nomogram failed to reach a high magnitude, this model showed significant higher discriminate ability compared with the AJCC or VALSG staging systems. Additionally, similar superiority was also found in the validation cohort when comparing the nomogram with the other two staging systems. It is notable that when stratifying the validation dataset into several different risk groups using the optimal cut-off values from the training cohort, significant distinctions were also observed in the survival curves, and even the risk groups were further categorized into different AJCC stages, which indicates the satisfied discriminate ability of this nomogram. Admittedly, in stage I patients, the differences were not significant in the survival curves for OS among different risk groups in the validation cohort, and there was also overlapping of survival curves in other stages. The restricted sample size may have contributed to this insignificance.

Based on a convenient scoring system, this prognostic nomogram provides clinicians with better guidance to identify high-risk patients with poor prognosis who may require additional treatment and intensive follow-up. However, our risk score system of different treatment modalities may not be appropriate for direct use, as the decision of treatment strategies involves multiple factors, not merely the TNM stages.

However, several limitations still exist in the present study. First, certain biases may exist due to the nature of this non-randomized, retrospective study. Second, certain weaknesses exist for using the SEER database, which provided only the crude mortality data and lacked in other routinely available parameters (e.g. performance score, smoking status, pulmonary functions, or body mass index) or any of the essential comorbidities (e.g. pulmonary hypertension, congestive heart failure, vascular disease, or renal failure). Dependency on the SEER database prevented us from including these parameters in this model. Moreover, the sequence of chemotherapy or radiotherapy with surgery was not considered, as the exact time for treatment was lacking in the SEER database. Consequently, we assumed chemotherapy or radiotherapy to be baseline variables instead of time-varying covariates; however, this assumption undoubtedly ignored the effects of treatment sequence on patients' survival. Hence, we will be conducting further multicenter research that incorporates relative completed clinicopathological variables, as well as detailed information regarding additional treatment, to refine the predictive power and generalizability of our model. It is hopeful that our nomogram model will create a more precise survival prediction when incorporating those unanalyzed parameters, which may include performance score, smoking status, pulmonary function, body mass index, and detailed additional treatment, as well as the above-mentioned comorbidities.

\section{CONCLUSIONS}

To date, no well-established tool has been reported for the prediction of survival in resected limited-stage SCLC patients. Our nomogram model was developed from integrated prognostic variables using a population-based US database, and externally validated in a Chinese cohort. This model consistently achieved appreciable prognostic ability, reliability, and clinical applicability, and hence may offer clinicians instructions for survival counseling, treatment strategy making, and clinical trial design. Furthermore, this proposed nomogram was also deployed into a website server for convenient application.

ACKNOWLEDGMENTS The authors thank all patients and institutions involved in this study, especially the ability to have open access to the SEER database.

FUNDING This work was supported the National Key Basic Research Development Plan (2018YFC1312105), the National Key Research and Development Program of China (2016YFC0901401), and the CAMS Innovation Fund for Medical Sciences (2016-I2M-1001, 2017-I2M-1-005).

DISCLOSURE Qingpeng Zeng, Jiagen Li, Fengwei Tan, Nan Sun, Yousheng Mao, Yushun Gao, Qi Xue, Shugeng Gao, Jun Zhao, and Jie He have no conflicts of interest to declare.

OPEN ACCESS This article is licensed under a Creative Commons Attribution 4.0 International License, which permits use, sharing, adaptation, distribution and reproduction in any medium or format, as long as you give appropriate credit to the original author(s) and the source, provide a link to the Creative Commons licence, and indicate if changes were made. The images or other third party material in this article are included in the article's Creative Commons licence, unless indicated otherwise in a credit line to the material. If material is not included in the article's Creative Commons licence and your intended use is not permitted by statutory regulation or exceeds the permitted use, you will need to obtain permission directly from the copyright 
holder. To view a copy of this licence, visit http://creativecommons. org/licenses/by/4.0/.

\section{REFERENCES}

1. Rudin CM, Ismaila N, Hann CL, et al. Treatment of small-cell lung cancer: American society of clinical oncology endorsement of the American college of chest physicians guideline. J Clin Oncol. 2015;33(34):4106-11.

2. Kalemkerian GP, Loo BW, Akerley W, et al. NCCN Guidelines Insights: Small Cell Lung Cancer, Version 2.2018. J Natl Compr Canc Netw. 2018;16(10):1171-82.

3. Fox W, Scadding JG. Medical research council comparative trial of surgery and radiotherapy for primary treatment of small-celled or oat-celled carcinoma of bronchus: ten-year follow-up. Lancet. 1973;2(7820):63-5.

4. Lad T, Piantadosi S, Thomas P, Payne D, Ruckdeschel J, Giaccone $\mathrm{G}$. A prospective randomized trial to determine the benefit of surgical resection of residual disease following response of small cell lung cancer to combination chemotherapy. Chest. 1994;106 (6 Suppl):320S-3S.

5. Yang CJ, Chan DY, Speicher PJ, et al. Surgery versus optimal medical management for N1 small cell lung cancer. Ann Thorac Surg. 2017;103(6):1767-72.

6. Yang CJ, Chan DY, Shah SA, et al. Long-term survival after surgery compared with concurrent chemoradiation for nodenegative small cell lung cancer. Ann Surg. 2018;268(6):1105-12.

7. National Comprehensive Cancer Network website. National Comprehensive Cancer Network Guidelines for small cell lung cancer version 2.2018. Available at: https://www.nccn.org/profe ssionals/physician_gls/pdf/sclc.pdf. Accessed 17 Jan 2018.

8. Jhun BW, Lee KJ, Jeon K, et al. Clinical applicability of staging small cell lung cancer according to the seventh edition of the TNM staging system. Lung Cancer. 2013; 81(1):65-70

9. Groome PA, Bolejack V, Crowley JJ, et al. The IASLC Lung Cancer Staging Project: validation of the proposals for revision of the $\mathrm{T}, \mathrm{N}$, and $\mathrm{M}$ descriptors and consequent stage groupings in the forthcoming (seventh) edition of the TNM classification of malignant tumours. J Thorac Oncol. 2007;2(8):694-705.

10. Abedallaa N, Tremblay L, Baey C, et al. Effect of chemotherapy in patients with resected small-cell or large-cell neuroendocrine carcinoma. J Thorac Oncol. 2012;7(7):1179-83.

11. Che K, Shen H, Qu X, et al. Survival outcomes for patients with surgical and non-surgical treatments in stages I-III small-cell lung cancer. J Cancer. 2018;9(8):1421-9.

12. Wong AT, Rineer J, Schwartz D, Schreiber D. Assessing the impact of postoperative radiation therapy for completely resected limited-stage small cell lung cancer using the national cancer database. J Thorac Oncol. 2016;11(2):242-8.
13. Xie D, Marks R, Zhang M, et al. Nomograms predict overall survival for patients with small-cell lung cancer incorporating pretreatment peripheral blood markers. $J$ Thorac Oncol. 2015;10(8):1213-20.

14. Pan H, Shi X, Xiao D, et al. Nomogram prediction for the survival of the patients with small cell lung cancer. J Thorac Dis. 2017;9(3):507-18.

15. Xiao HF, Zhang BH, Liao XZ, et al. Development and validation of two prognostic nomograms for predicting survival in patients with non-small cell and small cell lung cancer. Oncotarget. 2017;8(38):64303-16.

16. Wang S, Yang L, Ci B, et al. Development and validation of a nomogram prognostic model for SCLC patients. $J$ Thorac Oncol. 2018;13(9):1338-48.

17. Rami-Porta R, Bolejack V, Giroux DJ, et al. The IASLC lung cancer staging project: the new database to inform the eighth edition of the TNM classification of lung cancer. J Thorac Oncol. 2014;9(11):1618-24.

18. Abdel-Rahman O. Validation of the AJCC 8th lung cancer staging system among patients with small cell lung cancer. Clin Transl Oncol. 2018;20(4):550-6.

19. Liang W, Zhang L, Jiang G, et al. Development and validation of a nomogram for predicting survival in patients with resected nonsmall-cell lung cancer. J Clin Oncol. 2015;33(8):861-9.

20. Balachandran VP, Gonen M, Smith JJ, DeMatteo RP. Nomograms in oncology: more than meets the eye. Lancet Oncol. 2015;16(4):e173-80.

21. Yao Y, Zhou Y, Yang Z, Huang H, Shen H. Adjuvant chemotherapy following surgical resection improves survival in patients with early stage small cell lung cancer. Oncol Res. 2019;27(2):203-10.

22. Wakeam E, Acuna SA, Leighl NB, et al. Surgery versus chemotherapy and radiotherapy for early and locally advanced small cell lung cancer: a propensity-matched analysis of survival. Lung Cancer. 2017;109:78-88.

23. Takei H, Kondo H, Miyaoka E, et al. Surgery for small cell lung cancer a retrospective analysis of 243 patients from Japanese lung cancer registry in 2004. J Thorac Oncol. 2014;9(8):1140-5.

24. Zhao X, McCutcheon JN, Kallakury B, et al. Combined small cell carcinoma of the lung: is it a single entity? J Thorac Oncol. 2018;13(2):237-45.

25. Yokouchi H, Ishida T, Yamazaki S, et al. Prognostic impact of clinical variables on surgically resected small-cell lung cancer: results of a retrospective multicenter analysis (FIGHT002A and HOT1301A). Lung Cancer. 2015;90(3):548-53.

Publisher's Note Springer Nature remains neutral with regard to jurisdictional claims in published maps and institutional affiliations. 